Observations of electric discharge streamer propagation and capillary oscillations on the surface of air bubbles in water

This article has been downloaded from IOPscience. Please scroll down to see the full text article.

2011 J. Phys. D: Appl. Phys. 44082001

(http://iopscience.iop.org/0022-3727/44/8/082001)

View the table of contents for this issue, or go to the journal homepage for more

Download details:

IP Address: 141.211.173.82

The article was downloaded on 06/04/2012 at 16:54

Please note that terms and conditions apply. 


\title{
Observations of electric discharge streamer propagation and capillary oscillations on the surface of air bubbles in water
}

\author{
B S Sommers ${ }^{1}$, J E Foster ${ }^{1}$, N Yu Babaeva ${ }^{2}$ and Mark J Kushner ${ }^{2}$ \\ ${ }^{1}$ Department of Nuclear Engineering and Radiological Science, University of Michigan, Ann Arbor, MI, \\ 48109, USA \\ ${ }^{2}$ Department of Electrical Engineering and Computer Science, University of Michigan, Ann Arbor, MI, \\ 48109, USA
}

Received 15 November 2010, in final form 2 December 2010

Published 10 February 2011

Online at stacks.iop.org/JPhysD/44/082001

\begin{abstract}
The propagation of electric discharge streamers inside bubbles in liquids is of interest for the remediation of toxins in water and plasma-based surgical instruments. The manner of streamer propagation has an important influence on the production of reactive species that are critical to these applications. Streamer propagation along the surface of electrode-attached bubbles of air in water, previously predicted by numerical simulations, has been experimentally imaged using a fast frame-rate camera. The successive pulsing of the streamer discharge inside the bubbles produced oscillations along the air-water interface. Subsequent streamers were observed to closely follow surface distortions induced by such oscillations. The oscillations likely arise from the non-uniform perturbation of the bubble driven by the electric field of the streamer and were found to be consistent with Kelvin's equation for capillary oscillations. For a narrow range of applied voltage pulse frequencies, the oscillation amplitude increased over several pulse periods indicating, potentially, resonant behaviour. We also observed coupling between bubbles wherein oscillations in a second bubble without an internal discharge were induced by the presence of a streamer in a fixed bubble.
\end{abstract}

(Some figures in this article are in colour only in the electronic version)

\section{Introduction}

The production of plasmas in liquid water is of interest as an advanced oxidation technique in a variety of technological areas $[1,2]$. These applications range from the decomposition of volatile organic compounds (VOCs) and dyes in waste water to the development of point-of-use water purification [3-6]. The majority of these applications involve the formation of a gas phase electric discharge streamer within the liquid, which acts as a source of radicals, charged particles, UV light and shock waves that together comprise the advanced oxidation capacity of these systems $[7,8]$. One method under investigation for plasma formation in liquid water is to sustain the discharge within gas bubbles injected into the liquid [9]. Depending on the temperature and residence time of the bubbles, the gas composition ranges from that of the injected gas to the vapour pressure of the water, which consequently influences the reactive products formed by the discharge. The diffusion of these reaction products to the bubble interface and ultimately into the liquid water forms the source of improved reactivity of these systems. A more complete understanding of the dynamics of electric discharge streamer behaviour, propagation in bubbles and subsequent radical formation is important to the implementation of electric discharges for the 


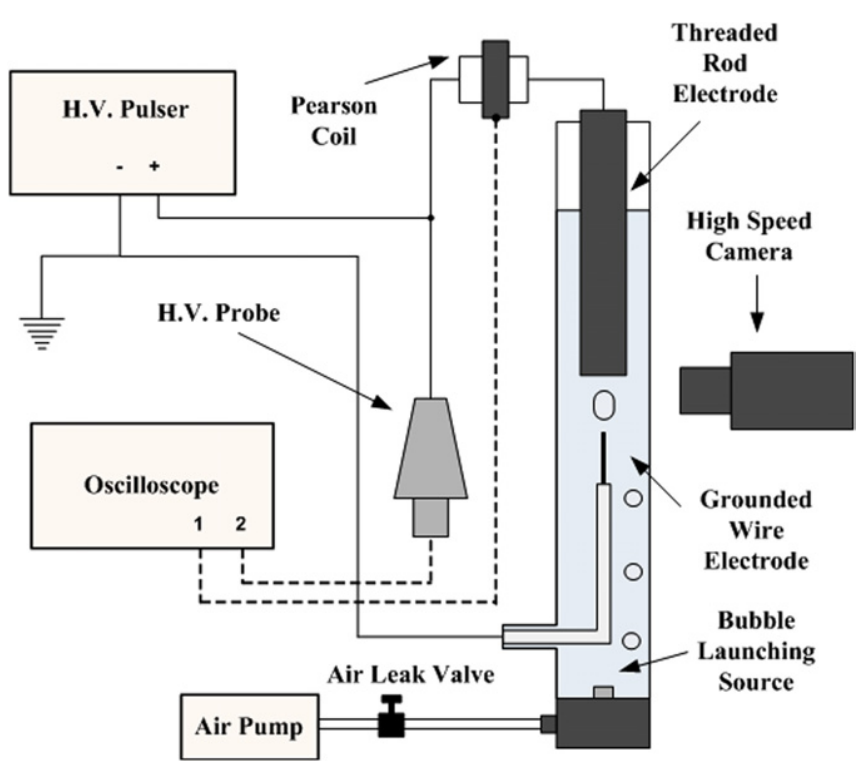

Figure 1. Schematic of the experiment. Bubbles pass between the electrodes and, in some cases, are trapped on the surface of the HV pulsed electrode.

treatment and purification of liquid water. If the streamer propagates along the air-water interface, which under certain conditions has been demonstrated experimentally [10] and predicted numerically [11], then the resulting reactive species will be in direct contact with the liquid, leading, potentially, to a more effective treatment of the liquid medium. In such numerical simulations, it has been shown that propagation along the air-water boundary will preferentially occur if the ratio of the dielectric constant of the liquid medium to that of the bubble vapour is large (for water this is 80).

In this paper, we discuss results from an experimental and computational investigation of the propagation of streamers in air bubbles submerged in water. We present observations of streamers propagating along the surface of bubbles and corroborating results from numerical simulations. We also discuss observations of streamer-excited capillary waves propagating along the air-water boundary, likely resulting from the interaction of the streamer with the bubble surface. Under certain conditions, these oscillations were observed to grow to large amplitudes over the course of many discharge pulses and oscillation cycles, indicating resonant growth. Similar oscillations were induced in neighbouring bubbles that did not have discharges within them.

The experiment and model are described in sections 2 and 3 , followed by a discussion of experimental observations of streamers in bubbles in section 4. Concluding remarks are in section 5 .

\section{Description of the experiment}

The primary experimental goal of this investigation was to image the interaction of streamers with the surface of an air bubble in liquid water. A schematic of the experimental setup is shown in figure 1. A pump was used to inject air bubbles into a small opening at the base of a water-filled
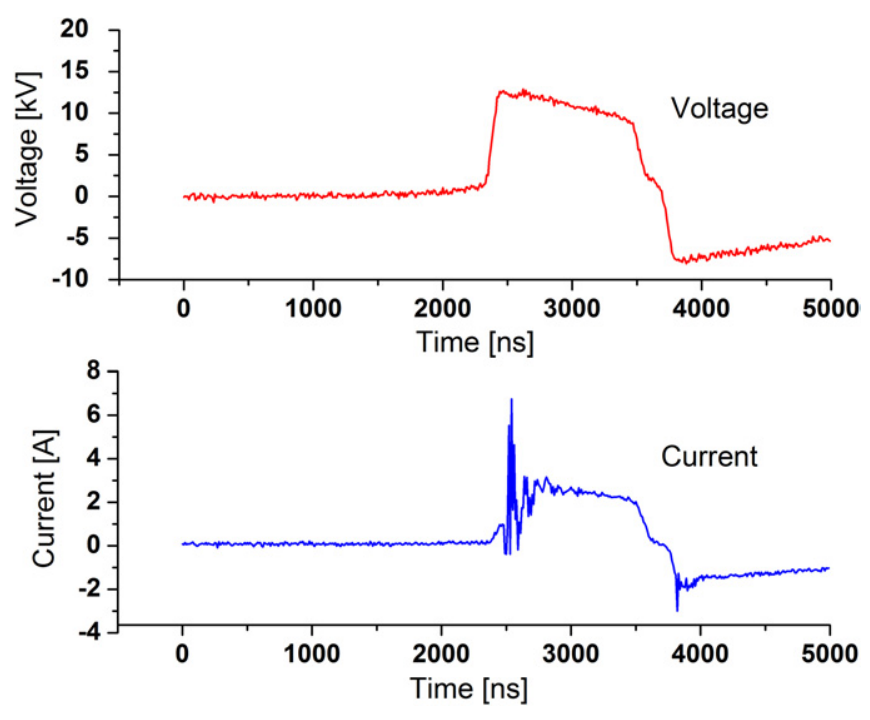

Figure 2. Discharge characteristics for $1 \mu$ s pulses applied at frequencies of 100-750 Hz. Voltage (top) and current (bottom) are shown.

glass tube with inner diameter $0.6 \mathrm{~cm}$ and dielectric constant of approximately 4. The gas was ambient room air. As the bubbles rise they pass between the electrodes. The electrodes consist of a grounded molybdenum wire $0.05 \mathrm{~cm}$ in diameter and a biased threaded rod $0.48 \mathrm{~cm}$ in diameter. The wire is placed approximately in the centre of the tube $0.4 \mathrm{~cm}$ below the biased rod. Typical bubble sizes ranged from 1 to $3 \mathrm{~mm}$ in radius. The larger bubbles were trapped at the bottom of the threaded rod. The water used was deionized water with a conductivity of $0.07 \mu \mathrm{S} \mathrm{cm}^{-1}$. A power modulator was used to apply high voltage pulses over a frequency range between 100 and $750 \mathrm{~Hz}$, with pulse rise times of $100 \mathrm{~ns}$ and pulse lengths of $1000 \mathrm{~ns}$. Voltage and current signals were measured using a $500 \mathrm{MHz}$ oscilloscope along with a Tektronix P6015A high voltage probe and a 6600 Pearson current transformer coil. A typical voltage and associated current pulse (at $500 \mathrm{~Hz}$ repetition rate) are shown in figure 2. Imaging was performed using a Redlake high speed camera operating at an acquisition frame rate of 5000 frames s$^{-1}$. The exposure time per frame was $197 \mu \mathrm{s}$.

\section{Description of the model}

The model used in this investigation, nonPDPSIM, is the same as that described in [11], and so will be only briefly discussed here. nonPDPSIM is a two-dimensional simulation in which Poisson's equation for the electric potential and transport equations for charged and neutral species are solved. The electron temperature, $T_{\mathrm{e}}$, is obtained by solving an electron energy conservation equation with transport and rate coefficients coming from local solutions of Boltzmann's equation. Radiation transport and photoionization are included by implementing a Green's function propagator. The numerical grid uses an unstructured mesh with triangular elements and refinement regions to resolve the details of the electrode tip and the bubble. The mesh consisted of 7400 nodes of which 4300 were in the plasma zone with tight refinement 

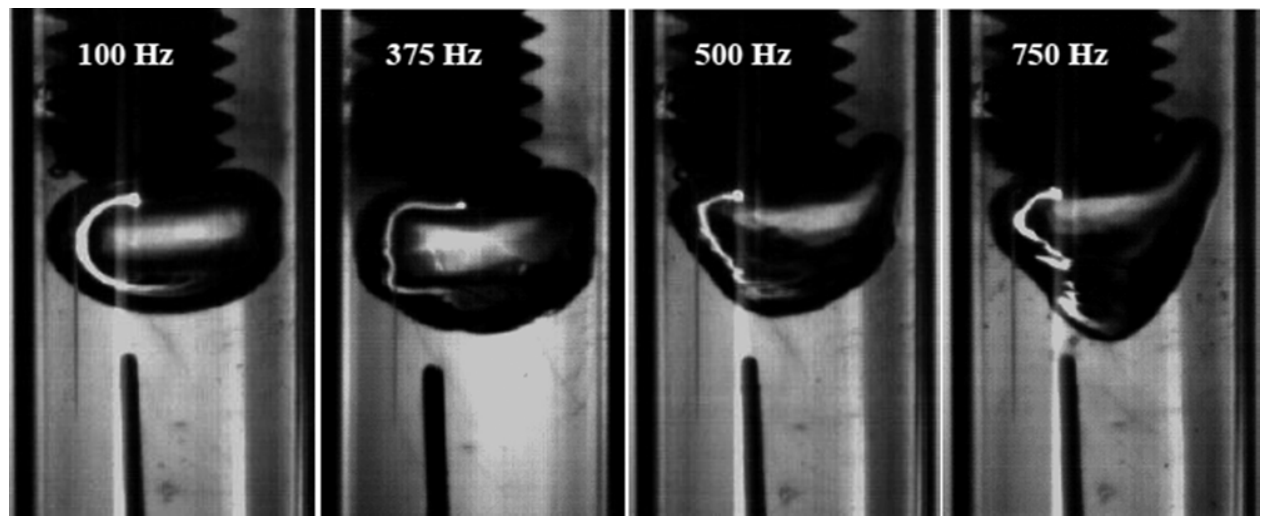

Figure 3. Streamers inside air bubbles in water are shown for pulse frequencies of $100-750 \mathrm{~Hz}$. The streamer follows the contour of the bubble surface even when there are inhomogeneities.

along the water-bubble interface. The plasma transport equations are solved only inside the gas bubble. Inside the liquid, a conductivity, $\sigma$, is specified and charge densities are solved for by $\partial \rho / \partial t=-\nabla \cdot \vec{j}=-\nabla \cdot \sigma \vec{E}$. Charge is allowed to accumulate at the bubble-surface interface consistent with the incident fluxes from the plasma, the conductivity of the liquid and the currents through the liquid. The gas mixture was $\mathrm{N}_{2} / \mathrm{O}_{2} / \mathrm{H}_{2} \mathrm{O}=79 / 18 / 3$ with an ambient pressure of $1 \mathrm{~atm}$ and the mean free path of ionizing photons was $100 \mu \mathrm{m}$. The discharge was initiated with a small cloud of seed electrons with a radius of $500 \mu \mathrm{m}$ and a peak density of $10^{8} \mathrm{~cm}^{-3}$ placed at the edge of the upper electrode. The bubble was static during the simulation.

\section{Imaging of streamers in bubbles}

In the case of water, which has a dielectric constant of 80, numerical simulations predicted that streamers will propagate along the air-water interface rather than through the shorter, more direct path within the volume of the bubble [11]. This indirect path is associated with electric field refraction at the boundary between materials with dissimilar dielectric constants. Four representative cases of streamer surface propagation in submerged bubbles pulsed at different frequencies are shown in figure 3 . The peak voltage is positive $12 \mathrm{kV}$. In each case, the streamer appears to travel along the surface of the bubble, terminating at some location on the bubble's bottom surface or slightly beyond the nadir. The streamer appears to follow the contours of the surface even when the bubble exhibits surface distortions, which become increasingly more violent from 375 to $750 \mathrm{~Hz}$ repetition rates. From these observations, the streamers, along with any resulting reaction products, appear to have direct contact with the liquid medium. Results from the model for streamer propagation in an electrode-attached bubble with similar geometry to the experiment are shown in figures 4 and 5 . In these simulations, a positive corona discharge was initiated at the corner of the electrode with a small spot of plasma. The electron density is shown in figure 4 for a smooth and wavy bubble during the $8 \mathrm{~ns}$ required for the streamer to traverse two-thirds of the bubble circumference. The corresponding positive charge density is shown in figure 5 . Consistent with

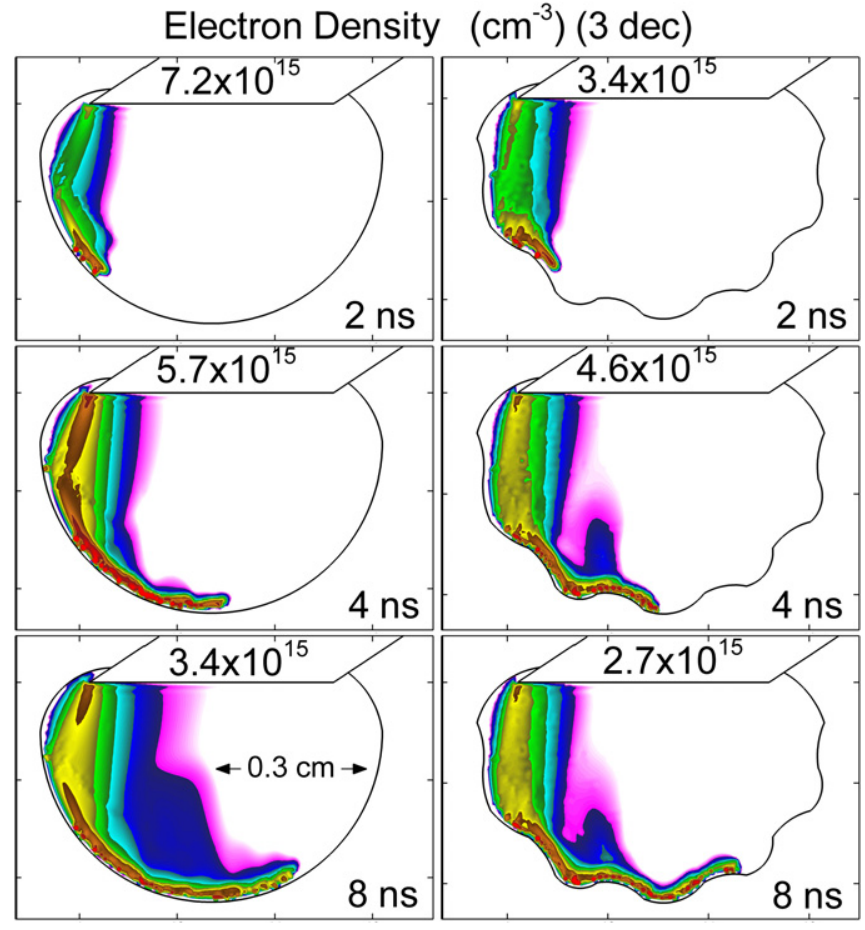

Figure 4. Predictions for electron density during streamer propagation as a function of time inside (left) smooth bubbles and (right) wavy bubbles. The contours are plotted on a 3-decade log scale with the maximum value shown in each frame.

experimental observations, the streamer travels along the airwater boundary, even after the introduction of distortions on the bubble surface. The maximum electron density reaches (4-7) $\times 10^{15} \mathrm{~cm}^{-3}$. The large electric field along the boundary (see discussion below) produces electron temperatures of 5-7 eV, which produce large radical densities along the airwater boundary. For example, the maximum density of $\mathrm{O}$ radicals produced is $6 \times 10^{16} \mathrm{~cm}^{-3}$ and that of $\mathrm{OH}$ radicals is $8 \times 10^{14} \mathrm{~cm}^{-3}$.

The propagation of the streamer along the gas-water interface is analogous to that of a streamer intersecting the dielectric in a dielectric barrier discharge. When the vertically oriented streamer first strikes the surface of the bubble, charge is deposited on the surface which generates lateral electric 


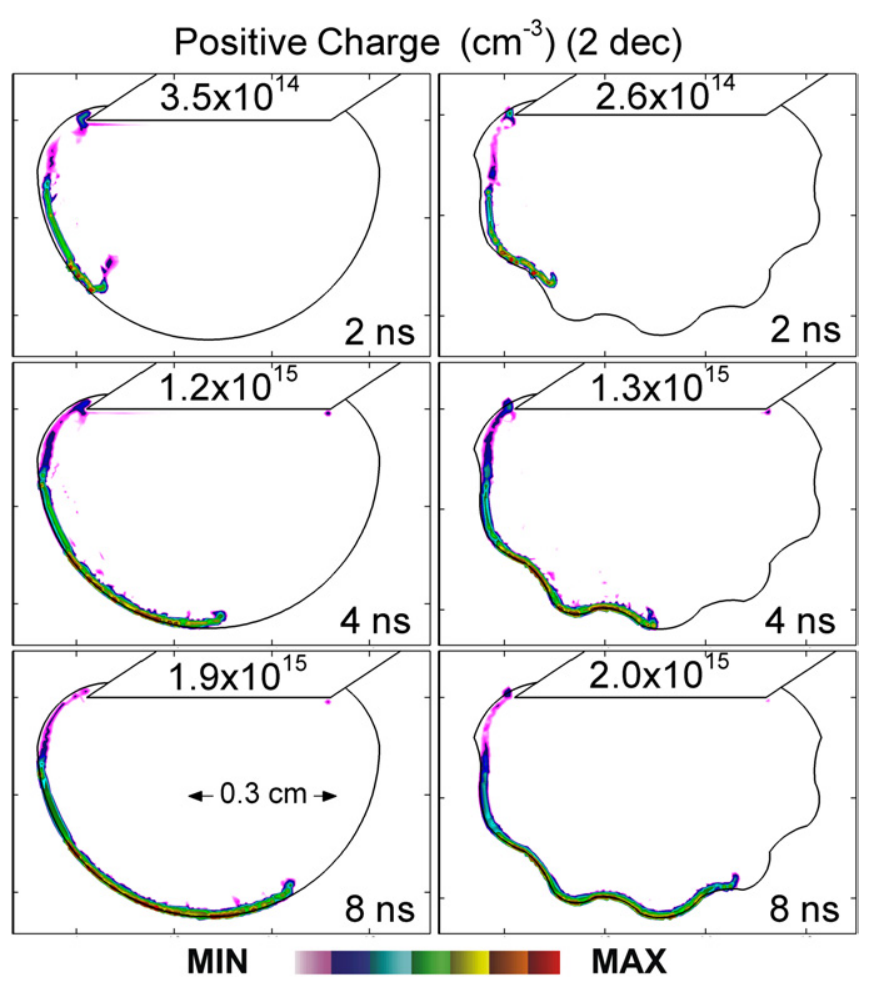

Figure 5. Predictions for positive charge density during streamer propagation as a function of time inside (left) smooth bubbles and (right) wavy bubbles. The contours are plotted on a 2-decade log scale with the maximum value shown in each frame.

fields which deflect the streamer parallel to the boundary. In this geometry, there are also components of the vacuum electric field that are directed parallel to the surface. The surface charging, as shown in figure 5, reinforces those parallel components. The gradient in dielectric constant across the bubble-water interface results in electric field enhancement, which further intensifies the avalanche. The production of components of the electric field parallel to the surface due to charging can be strong enough that the streamer propagates in a direction opposite to that of an unperturbed streamer. Under these conditions, an unperturbed streamer would propagate nearly in the direction of the vacuum electric field, which is downwards. The surface-hugging streamer passes beyond the nadir of the bubble and back towards the electrode opposite the unperturbed streamer due to the parallel electric fields along the surface produced by charging. It is for this reason that the streamer hugs the boundary in the wavy bubble, which directs the streamer vertically upwards at times.

The surface distortions shown in figure 3 appear to be a result of the streamer's interaction with the liquid-air boundary. This interaction may be rooted in the electrical stress created by the electric field at the tip of the streamer. The electrical stress distorts the surface of the bubble when the electrical pressure is comparable to the inward restorative force of the bubble's surface tension. The magnitude of the distortion scales with the electrical Weber number [12],

$$
W_{E}=\frac{\varepsilon_{0} E^{2} R_{0}}{\sigma}
$$

where $E$ is the magnitude of the electric field, $R_{0}$ is the radius of curvature of the surface and $\sigma$ is the surface tension. The larger $W_{E}$, the larger the propensity for the electric field to distort the surface. It is well known that the electric field at the tip of the streamer can be many times the average applied electric field or the critical field (Meeks condition) for breakdown in air $\left(\approx 30 \mathrm{kV} \mathrm{cm}^{-1}\right)[13,14]$. The electric field at the streamer head can reach up to hundreds of $\mathrm{kV} \mathrm{cm}^{-1}$ corresponding to $E / N$ exceeding $1000 \mathrm{Td}\left(1 \mathrm{Td}=10^{-17} \mathrm{~V} \mathrm{~cm}^{-2}\right)$ [15]. For example, the results of the model predict that the electric field at the tip of the streamer when it strikes the bubble surface is $510 \mathrm{kV} \mathrm{cm}^{-1}$ or $2100 \mathrm{Td}$. For $\sigma=7.17 \times 10^{-2} \mathrm{~N} \mathrm{~m}^{-1}$ and $R_{0}=3.0 \mathrm{~mm}$, this electric field produces $W_{E} \approx 970$, leading to the possibility of distortion of the surface of the bubble. As the streamer propagates along the surface, the electric field decreases as voltage is dropped along the streamer channel. Roughly half-way around the bubble, the electric field at the tip of the streamer is predicted from the model to be about $365 \mathrm{kV} \mathrm{cm}^{-1}\left(1500 \mathrm{Td}, W_{E} \approx 490\right)$ and when the streamer terminates, the electric field is about $185 \mathrm{kV} \mathrm{cm}^{-1}(770 \mathrm{Td}$, $W_{\mathrm{E}} \approx 130$ ). The charge deposition by the streamer on the surface of the bubble, as predicted by simulation, may also play a role in the distortion of the surface. There will be some component of repulsion of surface elements due to the positive charging. The rigidity of solid surfaces in conventional DBDs resists distortion by these positive charges, whereas the liquid surface may in fact become distorted.

The observed distortions of the surface may produce capillary waves, which propagate along the surface of the liquid-air boundary. Excitation of low amplitude waves on the liquid-air boundary is used as a means of measuring surface tension [16]. The propagation of such waves can be described in terms of $\sigma$, acceleration due to gravity $g$ and the liquid density $\rho$. This relation, known as Kelvin's equation, can be modified in the presence of a uniform electric field to yield [17]

$$
\omega^{2}=\frac{\sigma k^{3}}{\rho}+g k-\frac{\varepsilon_{0} E^{2} k^{2}}{\rho}
$$

where $\omega$ is the radian frequency of the capillary wave and $k$ is its wave number.

The durations of both the discharge pulse, only a few ns, and the applied electric field, $1000 \mathrm{~ns}$, are much less than the oscillation period $(\approx 4 \mathrm{~ms})$. It is expected that charge relaxation by the conductivity of the water would tend to eliminate residual surface charge on time scales shorter than the oscillation period. For liquid water the charge relaxation time is determined by the permittivity, $\varepsilon$, and conductivity, $\kappa$,

$$
\tau=\frac{\varepsilon}{\kappa} .
$$

For deionized water, $\tau \approx 100 \mu \mathrm{s}$, which is much shorter than the oscillation period. The electric field due to both the streamer and charge deposition will therefore act as a fast impulse that excites the bubble surface but is not present during the propagation of the wave. For this reason, the electric field in equation (2) can be neglected at later times $(t>100 \mathrm{~ns})$ after the pulse. Images of the bubble over half a cycle $(500 \mathrm{~Hz}$ repetition rate and peak voltage $12 \mathrm{kV}$ ) are shown in figure 6 . 

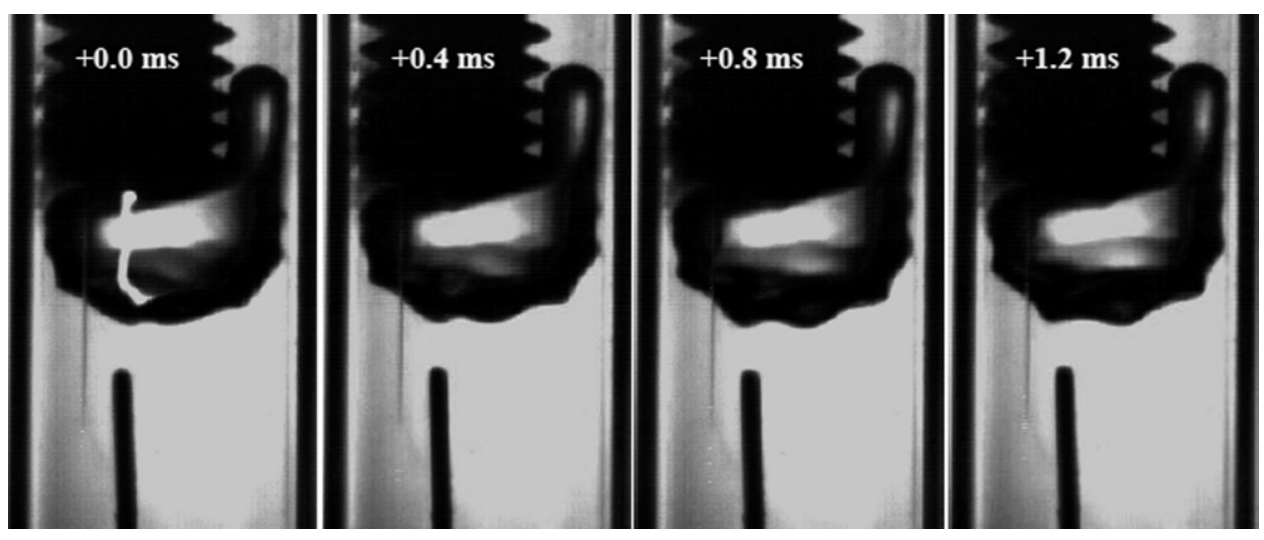

Figure 6. Images of a bubble as a function of time (noted in each frame relative to the discharge pulse) following a streamer striking the inside surface. The streamer imparts an impulse to a region of the bubble surface, leading to the excitation of capillary waves whose characteristics agree with Kelvin's equation.

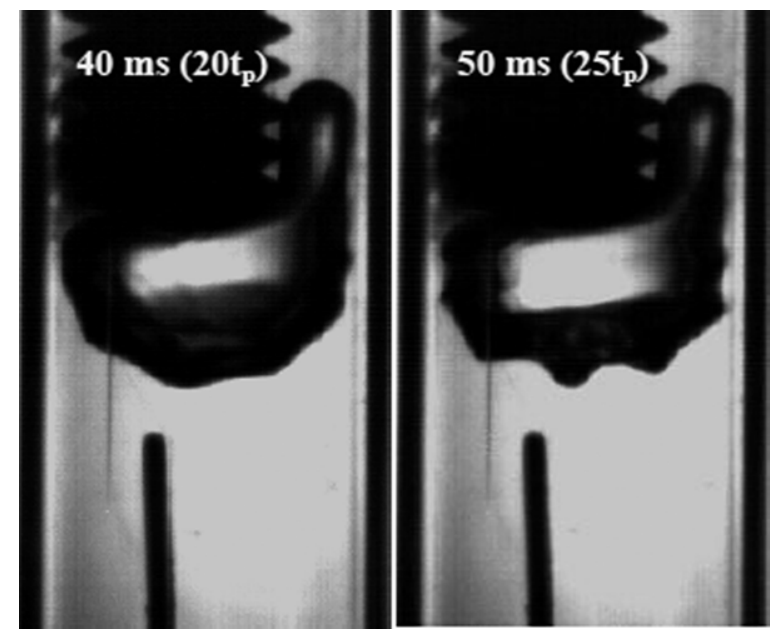

Figure 7. Images of bubble oscillations driven at $500 \mathrm{~Hz}(2 \mathrm{~ms}$ period) by streamer impacts. The time in each frame is relative to the first streamer impact (or number of pulse periods $t_{\mathrm{p}}$.) The surface wave growth may be related to resonant driving of the bubble surface.

The times noted in the figure are relative to the streamer impact. At $t=0 \mathrm{~ms}$, the streamer terminates on the bottom of the bubble resulting in exciting (or enforcing existing) surface oscillations at the bottom of the bubble, either exciting new surface oscillations or reinforcing pre-existing ones. The wavelengths of the surface oscillations are $1.6-1.9 \mathrm{~mm}$ and oscillation frequencies are approximately half of the applied pulse frequency $(500 \mathrm{~Hz})$. Kelvin's equation predicts that for wavelengths in this range, the frequency should be 250 $350 \mathrm{~Hz}$, which corresponds well with the observations.

At $500 \mathrm{~Hz}$, the oscillations grow to large amplitudes over the course of several discharge pulse cycles. The growth in amplitude of the bubble oscillations is shown in figure 7. The times indicated in the figure are relative to the first streamer impact and are also given as a multiple of the streamer pulse period $t_{\mathrm{p}}(2 \mathrm{~ms})$. As the bubble continues to be driven, its oscillation amplitude grows monotonically, beginning with what appears to be a single mode and eventually becoming multimode. The precise nature of this wave growth is likely related to the resonant nature of the streamer impact in this particular case. The frequency of the capillary waves $(\approx 250$ $350 \mathrm{~Hz}$ ) is nearly half of the driving frequency of the high voltage pulses $(500 \mathrm{~Hz})$, resulting in the streamer striking the bubble surface once every half oscillation cycle, but not necessarily at the same location. The impact site of the streamer appears to be somewhat random, preferentially striking the bubble during its expansion phase rather than during its contraction phase, presumably where $E / N$ is maximum. This tendency may be caused by a feedback mechanism from the already distorted shape of the bubble and surface charge, which in turn distorts the shape of the local field and perhaps creates a preferred path for the streamer. The resonant wave growth did not occur at other pulse frequencies investigated.

The propagation of the streamer-induced capillary wave oscillations couples to other bubbles. The interactions between two bubbles undergoing capillary wave oscillations for a pulse frequency of $500 \mathrm{~Hz}$ and peak voltage of $8.5 \mathrm{kV}$ are shown in figure 8. Times are shown relative to the impact of the streamer. In this case, the larger bubble, which is attached to the electrode, begins oscillating in response to the streamer pulses. When in close proximity, the smaller adjacent bubble manifests similar surface oscillations. The nature of this energy transfer is not fully understood, but the interaction is believed to be driven by the propagation of pressure disturbances from the attached bubble through the water. These pressure waves arrive at the second bubble and subsequently excite capillary wave oscillations on its surface. The two bubbles appear to oscillate $90^{\circ}$ out of phase from each other, which could result from a propagation delay. The wavelength on the surface of each bubble is shorter than for a single bubble by a factor of about two $(0.7-0.8 \mathrm{~mm})$, suggesting that a new mode is excited that is different from the case of a single bubble. The capillary oscillation frequencies for both bubbles, approximately 1000$1250 \mathrm{~Hz}$, are well above the repetition rate of the pulsed discharge. These frequencies are also consistent with those predicted by Kelvin's equation. From Kelvin's equation, the oscillation frequency for the observed wavelengths should be $950-1150 \mathrm{~Hz}$. 

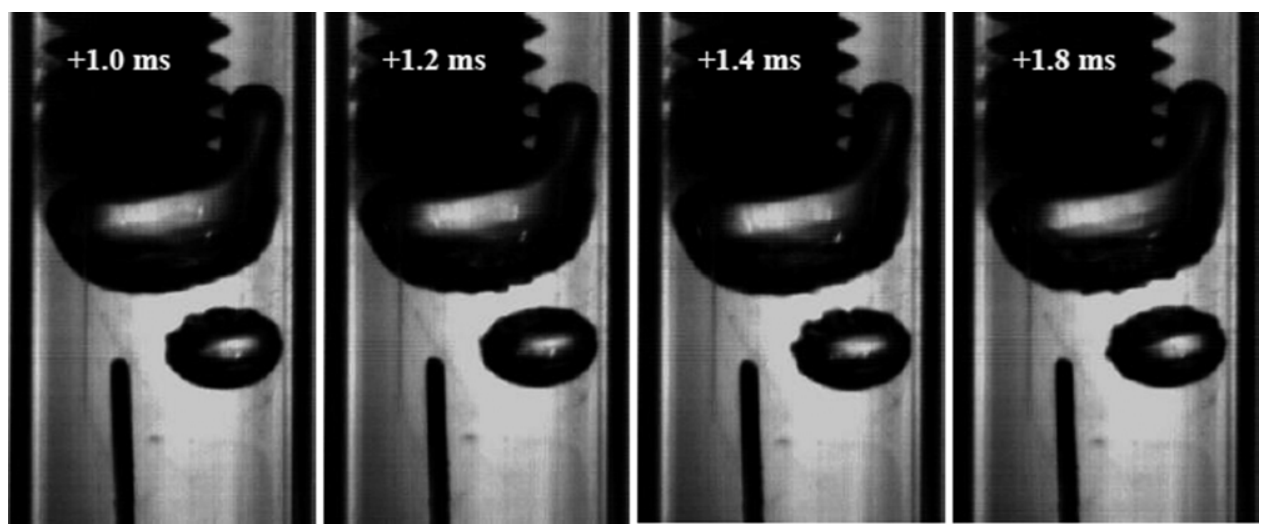

Figure 8. Images of apparent interactions and communication of capillary waves from bubble to bubble as a function of time relative to the first streamer (indicated in each frame).

\section{Concluding remarks}

The interactions of surface streamers in air bubbles in water were investigated using high speed imaging. The observation of streamer propagation along the surface of the bubbles agrees well with computer modelling. It was also observed that the streamers tended to excite waves at the liquid-gas interface of the bubbles. The wavelength and frequency of these oscillations were consistent with Kelvin's equation for capillary waves. The streamer-induced modification of the interface via capillary waves affects the local electric field, which in turn may provide feedback to streamer propagation. Depending on the excitation frequency of the streamers, these waves exhibited growth, indicating a resonant effect. The capillary oscillations produced in electrode-attached bubbles by streamers can be transmitted to free bubbles through pressure interactions.

\section{Acknowledgments}

This work was supported by the National Science Foundation (CBET 0939879) and the Department of Energy Office of Fusion Energy Sciences.

\section{References}

[1] Malik M Ghaffar, A and Malik 2001 Plasma Sources Sci. Technol. 1082
[2] Locke B R, Sato M, Sunka P, Hoffmann M J and Chang J S 2005 Indust. Eng. Chem. Res. 45882

[3] Nazerenko O B and Shubin B G 2006 Investigation of electric discharge treatment of water for ammonium nitrogen removal Proc. 2nd Environmental Physics Conf. (Alexandria, Egypt, 18-22 February 2006)

[4] Baroch P, Anita V, Saito N and Takai O 2008 J. Electrostat. 66294

[5] Yamabe C, Takeshita F, Miichi T, Hayashi N and Ihara S 2005 Plasma Process. Polym. 2246

[6] Foster J E, Weatherford B R, Gillman E and Yee B 2010 Plasma Sources Sci. Technol. 1925001

[7] Joshi R P, Kolb J F, Xiao S and Schoenbach K H 2009 Plasma Process. Polym 6763

[8] Sugiarto A, Ohshima T and Sato M 2002 Thin Solid Films 407174

[9] Bruggeman P and Leys C 2009 J. Phys. D: Appl. Phys. 42053001

[10] Bruggeman P 2007 J. Phys. D: Appl. Phys. 401937

[11] Babaeva N and Kushner M J 2009 J. Phys. D: Appl. Phys. 42132003

[12] Shaw S J and Spelt P D M 2009 Proc. R. Soc. A $4653127-42$

[13] Meek J M 1940 Phys. Rev. 57 722-8

[14] Babaeva N and Kushner M J 2009 Plasma Sources Sci. Technol. 18035009

[15] Nakamura S et al 2009 Optical measurements of the electric field of pulsed streamer discharges in water IEEE Trans. Dielectr. Insul. 161117

[16] Sohl C H, Miyano K and Ketterson J B 1978 Rev. Sci. Instrum. 491465

[17] Hayes C F 1975 J. Phys. Chem. 791689 